The Journal of Animal \& Plant Sciences, 30(6): 2020, Page: 1380-1388

ISSN (print): 1018-7081; ISSN (online): 2309-8694

\title{
SYNERGISTIC EFFECT OF EXTRACTS OF GINKGO BILOBA LEAF AND PANAX GINSENG ROOT ON CARBOHYDRATE AND LIPID METABOLISM GENE EXPRESSION IN ALLOXAN INDUCED DIABETIC RATS
}

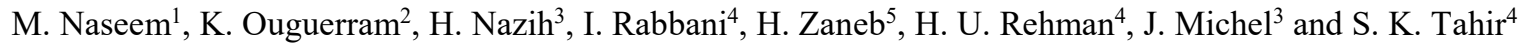 \\ ${ }^{1}$ Department of Zoology, University of Balochistan, Quetta- Pakistan \\ ${ }^{2}$ UMR 1280 Phan, INRA, CRNH, West Human Nutrition Research Center, CHU, Nantes F-44093, France \\ ${ }^{3}$ Faculty of Pharmacy, University of Nantes, Nantes- France \\ ${ }^{4}$ Department of Physiology, University of Veterinary and Animal Sciences, Lahore- Pakistan \\ ${ }^{5}$ Department of Anatomy and Histology, University and Animal Sciences, Lahore- Pakistan. \\ Corresponding Author's E-mail: mahrukhnaseem@rocketmail.com
}

\begin{abstract}
The present study aimed to evaluate the anti-diabetic properties of Ginkgo biloba leaves extract (GBE) and Panax ginseng roots extract (PGE) in different combinations. A total of 40 rats were fed on high-fat-diet for 14-days, then divided into five groups ( $\mathrm{N}=8$ ). Non diabetic group (NDG), Diabetic-group (DG), Mixed-group-1 (MG1), Mixed-group2 (MG2), Mixed-Group-3 (MG3). Alloxan monohydrate (120-130 mg/Kg BW) was used as a diabetogenic agent. The data of blood glucose and body weight (BW) were monitored regularly weekly. Basal blood was collected from the heart for biochemical analyses. Skeletal muscle, hepatic, and adipose tissue were obtained for mRNA expression of genes. A Significant decrease in BW was found in all treated groups. A significant reduction in fasting serum glucose, AST, ALT, and creatinine were recorded in dose dependent-manner. The treatments showed up-regulation of GLUT-4 in the muscle (all treated groups) and hepatic tissues (MG3); IR in the muscle (MG3) and adipose tissue (MG3), and IRS-1 in hepatic (MG3) and adipose tissue (MG3). Our results showed that these herbs improve dyslipidemia and have strong antioxidant activities. We found significant down-regulation for SREBP-1c in dose-dependent manner in the liver and significant upregulation for FAS (MG2 \& MG3) in the liver. Significant up-regulation was found for PPAR- $\alpha$ in muscles and PPAR- $\gamma$ in adipose tissues in all treated groups. Significantly down-regulation for TNF- $\alpha$ seemed in all studied organs. In conclusion, GBE and PGE showed strong anti-diabetic, anti-hypercholesterolemia and anti-oxidative effects in combination by regulating the genes involved in carbohydrate and lipids metabolism.
\end{abstract}

Key words: Alloxan, Diabetes, Ginkgo biloba leaf extract, Metabolism, Panax ginseng root extract, Rats.

https://doi.org/10.36899/JAPS.2020.6.0158

Published online August 03,2020

\section{INTRODUCTION}

Diabetes mellitus (DM) becoming a leading health problem in the last few decades and characterized by chronic hyperglycemia; associated with metabolic disturbance of carbohydrate, protein and fats; caused due to deregulation in the mechanism of insulin actions and its secretion, or even both (Naseem et al., 2016). Oxidative stress is a common problem in diabetes which may lead to neuropathy, retinopathy, nephropathy, cardiovascular disease and other major health complications (Krishnamurthy et al., 2011). There is strong evidence to support the fact that hyperglycemia elevated reactive oxygen species (ROS) generation (Lin et al., 2008; El-Karim et al., 2017). Enhanced ROS results in an imbalance between free radicals generations and anti-oxidative defense systems of the body, thus leading to the development of diabetic-associated complications (Kumar, 2012). Interest in the plants derived anti-hyperglycemic agents are gradually increased in the last few years due to its less adverse effects (Lee et al., 2010).

Ginkgo biloba (GB) is a well-known oldest tree species that still exists on the earth with certain health benefits (Naseem et al., 2016). Terpenoids (6-7\%) and flavonoids (34-26\%) are the active ingredients of ginkgo (Smith and Luo, 2003). Ginkgo is a dioecious tree belongs to Ginkgoaceae family, and is traditional Chinese herbal medicines used to treat diabetes, cancer, bronchitis, sexual dysfunction, chilblains, aging, Alzheimer's disorder, cerebral, peripheral and neuronal diseases (Unger, 2013; Kamel and Abd-Elrhman, 2018).

Panax ginseng is a perennial herb having fleshy root, belongs to Araliaceae family. Ginsenosides is the active component of this herb (Kim et al., 2014). Ginseng is another traditional Chinese herb has strong antiinflammatory, anti-fatigue, anti-aging, anti-oxidant and anti-apoptotic properties. Also use to treat diabetes, Central nervous system disorders, cardiac stroke, weak immunity and sexual dysfunction (Amin et al., 2011; Kim et al., 2014; Vuksan et al., 2019). 
To the best of our knowledge, no published data is available on the anti-diabetic properties of GBE and PGE in combination. The objective of the study was to analyze the anti-diabetic activities of GBE and PGE the molecular mechanisms of carbohydrates and lipid metabolism in diabetic rats. Furthermore, the positive role of these two natural remedies on hyperlipidemia, hypercholesterolemia and oxidative stress related to diabetes was also assessed.

\section{MATERIALS AND METHODS}

Experimental animals: Forty adult Wistar male rats (150-200 g); with two rats per cage were confined in a controlled environment " $24 \pm 5{ }^{\circ} \mathrm{C} ; 12 \mathrm{~h}$ light/dark cycle" with free water and food access in the animal facility of University of Veterinary and Animal Sciences, Lahore (UVAS), Pakistan, prior to the experiment. Body weight (BW) was recorded weekly during the study of 14 weeks. The experiment was performed according to guidance and recommendations of the institutional ethical committee for the care of Laboratory Animals (UVAS).

Experimental Design and Animal Grouping: Rats were fed on a high fat diet "HFD: $12.7 \%$ maize starch, $6 \%$ cellulose, $6.5 \%$ dextrose, $31 \%$ beef tallow, $28.6 \%$ casein, 3.9\% sun flower oil, 9.7\% minerals and 1.3\% vitamins by weight" for 14 days, and randomly divided into following five groups $(\mathrm{N}=8)$ :

- Non diabetic group (NDG): This group included non-diabetic rats (negative control), feed on the standard diet without herbal extract treatment.

- Diabetic-group (DG): This group included diabetic rats (positive control) and feed on the standard diet without herbal extract treatment.

- $\quad$ Mixed-group-1 (MG1): The diabetic rats were treated with the combination of GBE $(50 \mathrm{mg} /$ $\mathrm{kg} /$ day) and PGE (150 mg/ kg/ day) mixed in standard chow.

- $\quad$ Mixed-group-2 (MG2): The diabetic rats were treated with the combination of GBE (100 mg/ $\mathrm{kg} /$ day) and PGE (300 mg/ kg/ day of PGE) mixed in standard chow.

- $\quad$ Mixed-group-3 (MG3): The diabetic rats were treated with the combination of GBE $(150 \mathrm{mg}$ $/ \mathrm{kg}$ / day) and PGE (450 mg/ kg/ day) mixed in standard chow.

After 14 days, alloxan monohydrate (Sigma, USA) dissolved in $0.5 \mathrm{ml}$ saline and injected intraperitoneal as a single dose (120-130 mg/kg BW) in overnight fasting rats. Hypoglycemia was prevented by giving 20\% (for $6 \mathrm{~h}$ ) and 5\% (for next 24h) glucose solutions (Ebuehi et al., 2010). After $72 \mathrm{~h}$ from alloxan dosing, fasting blood glucose level was measured by tail puncture; rats having blood glucose level higher than 250 $\mathrm{mg} / \mathrm{dl}$ were selected for the experiment (Cheng et al., 2012; Naseem et al., 2016). Supplementation of the standardized GBE (terpenoids 6-7\% flavonoids 24-27\%) and PGE (ginsenosides 4\%) (Hunan Nutramax Inc, China) was started. Dosing was done by mixing both herbal extracts in different combinations in the animal diet for 14-weeks.

Blood collection and Serum biochemical analysis: After 14 weeks rats were kept in fasting state for 24 hours and then blood samples were taken directly from heart puncture. Serum was separated from blood by the method described by (Kumar, 2012) and kept at $-20^{\circ} \mathrm{C}$ for further analysis. The biochemical analyses of fasting serum glucose (FSG), malondialdehyde (MDA), catalase (CAT), Alanine Aminotransferase (ALT), Aspartate Aminotransferase (AST) and creatinine were performed by commercially available kits (Randox, UK). Total serum cholesterol (TC) and triglycerides (TG) were done by enzymatic kits (Bio-Merieux, France). Fast Protein Liquid Chromatography (FPLC USA) was used for lipoprotein profile (VLDL-c, LDL-c, HDL-c).

Skeletal muscle, hepatic and adipose tissues collection and their mRNA expression of genes determination: Rats were killed skeletal muscle, adipose and hepatic tissues were collected, washed with normal saline and kept at $-80^{\circ} \mathrm{C}$. The total RNA extraction from the frozen tissues was done by using trizol reagent (Ambion, USA) as per manufacturer's instructions. The quantification of total RNA was done by nanodrop spectrophotometer (absorbance at $260 \mathrm{~nm}$ ). Thereafter, about $1 \mu \mathrm{g}$ of total RNA was reverse-transcribed into complementary DNA (cDNA) using Super-Script-III reverse Transcriptase (Invitrogen, France) in a $20 \mu \mathrm{l}$ reaction volume. The denaturation step was carried out for $5 \mathrm{~min}$ at $70^{\circ} \mathrm{C}$, followed by an elongation for $45 \mathrm{~min}$ at $55^{\circ} \mathrm{C}$. Quantitative PCR was performed on a MyiQ2 RealTime-PCR (Bio Rad, Marnes-la-coquette, France) using SYBR Green (Bio Rad) supermix according to the manufacturer's instructions. The PCR was carried out for 45 cycles of $95{ }^{\circ} \mathrm{C}$ for $30 \mathrm{~s}$ and $60{ }^{\circ} \mathrm{C}$ for $30 \mathrm{~s}$ and $72^{\circ} \mathrm{C}$ for $30 \mathrm{~s}$. The fluorescence was read during the reaction, allowing continuous monitoring of amount of PCR product. Primers were designed through primer3 website. The GADPH was used as a house-keeping gene. The $2^{\Lambda} \Delta \Delta \mathrm{CT}$ method was used for relative quantification. Primers used for qRT-PCR are given in Table 1 .

Statistical analysis: Results represented as mean $\pm \mathrm{SEM}$. We used one way ANOVA followed by PLSD Fisher's test (Statview software; SAS Institute Inc, USA) to measure physiological and biochemical parameters while the data of mRNA genes expression were analyzed by Kruskal Wallis test, followed by Fisher's test. The $\mathrm{p}<0.05$ was considered as significant. 


\section{RESULTS AND DISCUSSION}

The prevalence of diabetes increasing globally at an alarming rate is attributed to a mordent lifestyle, high cholesterol diet and lack of physical activities. Pharmacological drugs for the treatment of diabetes cause a high risk of secondary health issues (Xie et al., 2002). Thus due to this risk, the therapeutic use of herbal remedies has been significantly increased worldwide. Since G. biloba and P. ginseng are widely used for the treatment of many disorders including diabetes, but we used both these natural remedies in combination. In the present study, we evaluated the synergistic effects of these two herbs on the metabolic disturbance associated with alloxan induced diabetic rat model. To the best of our knowledge, no data is available as an anti-diabetic effect of GBE and PGE in combination, so we discussed all the parameters separately for GBE and PGE throughout the text.

Body weight: The BW was measured weekly; however, data of the $1^{\text {st }}$ and $14^{\text {th }}$ week is presented here (Table 2). Significant reduction $(\mathrm{p}<0.0001)$ in BW from week-1 to week-14 was found in diabetic rats, however, all treated groups showed suppression in BW reduction. The results for alloxan-induced diabetic rats are according to other authors (Lee et al., 2012; Cheng et al., 2012; Naseem et al., 2016). In diabetes, decrease in BW might be due to improper protein, lipids or glucose metabolisms (Baynes et al., 1991), tissue proteins degradation (Swanston-Flatt et al., 1990), adipocytes degeneration, excessive conversion of glycogen to glucose by muscle and frequent micturition (Ramadan et al., 2009).

Blood glucose concentration and mRNA gene expression of carbohydrate metabolism: Impaired glucose metabolism is a characteristic feature of diabetes. We evaluated that GBE and PGE contain hypoglycemic properties, since, all treated groups in this study indicated a significant $(\mathrm{p}<0.0001)$ decrease in blood glucose level (Table 2). Furthermore, fasting serum glucose (FSG) levels were also measured at the end of the trial. Significant $(p<0.0001)$ increase in FSG concentration was noted for DG however; significant $(p<0.0001)$ reduction was found in all the treated groups (Table 3 ). Previously, many researchers studied the antihyperglycemic effects of GBE and PGE separately. Impaired glucose tolerance is a characteristic feature of diabetes. Untreated prolonged hyperglycemia may cause $\beta$-cells toxicity which leads to enhanced blood glycation thus damages various body tissues/organs (Alam et al., 2014).

To know the underlying mechanism, how GBE and PGE reduce blood glucose concentration we also performed mRNA gene expression involved in carbohydrate metabolism (GLUT-4, IR and IRS-1). In muscles, significant up-regulation was observed for
GLUT-4 $(\mathrm{p}<0.0001)$ in all treated groups (Figure 1) and MG3 $(\mathrm{p}<0.05)$ in the liver (Figure 2$)$, which previously found to be down-regulated in DG. No significant results were found for GLUT-4 in adipose tissues (Figure 3). Results for ginkgo (Naseem et al., 2016) and ginseng (Jeon et al., 2013; Cheon et al., 2015) are in agreement with others. Glucose utilization by skeletal muscles is attributed to GLUT-4 which itself regulated by blood insulin concentration (Leto and Saltiel, 2012). The primary storage site for both glycogen and glucose in skeletal muscles and also oxidized these molecules to release an adequate amount of energy. Glucose metabolism is regulated by removing excess of glucose from the circulatory blood via various glucose transport channels/mechanisms like GLUT-4 (Huang and Czech, 2007).

We found significant up-regulation for IR in MG3 both in skeletal muscles $(\mathrm{p}<0.0001)$ (Figure 1) and adipose tissues $(\mathrm{p}<0.05)$ (Figure 3$)$, however, no significant data was found for hepatic tissue (Figure 2). Insulin is the primary factor to regulate glucose uptake by body tissues via a complex cascade signaling pathway. Insulin binds with IR to carry out its various cellular activities to regulate glucose metabolism. Thus any impairment in IR might lead to the disturbance of insulin efficacy and develops insulin resistance (Calle et al., 2008). For IRS-1 significant down regulation was found in the diabetic group. However, significant upregulation was noted in MG3 both for the liver $(\mathrm{p}<0.05)$ (Figure 2) and adipose tissues $(\mathrm{p}<0.0001)$ (Figure 3$)$. IRS-1 plays a vital role in transduction intracellular insulin signals. Thus, if intracellular insulin concentration is enhanced IRS-1 can up-regulate its expression (Zhou et al., 2011). Since hepatic insulin resistance is a key factor for diabetes development. Hepatic insulin resistance linked with the down-regulation of IRS-1 and IRS-2 (Taniguchi et al., 2005). Thus regulation of GLUT-4, IR and IRS-1 in our study indicated that GBE and PGE help to improve insulin cell sensitivity and thus regulate glucose utilization by tissues/muscles.

Serum lipid Profile and mRNA gene expression of lipid/fat metabolism: Dyslipidemia and hypertriglyceridemia are important factors of cardiovascular disorders in diabetes. In diabetes increased lipolysis, dysfunction in cholesterol packing and elevated plasma free fatty acid level is the primary reasons for hypercholesterolemia and hyper-triglyceridemia which leads to the development of cardiovascular disorders (Cho et al., 2006).

The results showed that these herbs in combination improve dyslipidemia and hypertriglyceridemia. A significant decrease for VLDL-c was found in MG1 $(p<0.05),(p<0.0001) M G 2$ and $(p<0.0001)$ MG3, a significant $(\mathrm{p}<0.0001)$ decrease for LDL-c serum level was also recorded in all treated groups. However, 
for HDL-c significant increase was recorded in MG1 $(p<0.001)$, both for MG2 and MG3 $(p<0.0001)$. A significant decrease for TG was recorded in all the three treated groups, however, we found a significant reduction for TC only in MG3 $(\mathrm{p}<0.001)$ (Table 3). Other researchers also found a significant increase in TC and TG blood levels in alloxan-induced diabetic rats (Ebuehi et al., 2010; Naseem et al., 2016).

We also try to evaluate how GBE and PGE regulate dyslipidemia in association with lipid metabolic genes (SREBP- $1 \mathrm{c}$, FAS, PPAR- $\alpha$, and PPAR- $\gamma$ ). SREBP $-1 \mathrm{c}$ was done only in hepatic tissues and we observed down-regulation in MG2 $(\mathrm{p}<0.05)$ and MG3 $(\mathrm{p}<0.001)$, which showed up regulation in diabetic rats (Figure 2). The liver is the main organ for fat and carbohydrate metabolism. Insulin regulates SREBP-1c; a lipid metabolic gene (Yuan et al., 2011). SREPB-1c regulates several genes that are involved in free fatty acid, phospholipids, TG and TC synthesis (Horton et al., 2002). The findings for ginkgo (Zhou et al., 2011) and ginseng (Lee et al., 2010) are in agreement with other researchers. In hepatic tissues, significant up-regulation was noted for FAS in MG2 and MG3 $(p<0.05)$, which previously showed down-regulation in DG (Figure 2), we observed no significant change for FAS in any of the treated group in adipose tissues (Figure 3). In liver; FAS catalyzed the biosynthesis of fatty acid (Menendez et al., 2009). However, diabetes markedly suppressed its expression in the liver (Naseem et al., 2016), as also found in our study.

In muscles, PPAR- $\alpha$ was found to be downregulated in diabetic condition and we measured significant up-regulated $(p<0.001)$ of this gene for all treated groups (Figure 1). No significant change was found for PPAR- $\alpha$ in hepatic tissues (Figure 2). PPAR- $\alpha$ regulates genes involved in lipoproteins and lipids metabolism binding with PPAR response elements. The PPAR- $\alpha$ along with apo AI and AII enhanced the HDL blood concentration. Thus dysfunctioning in the expression of PPAR- $\alpha$ distorts the fatty acid and lipoprotein metabolisms; in turn leads to the abnormal levels of blood TC and TG (Yoon et al., 2003).

The PPAR- $\gamma$ was done only in adipose tissues and we found significant up-regulation in MG1 and MG2 $(p<0.001)$ and MG3 $(p<0.0001)$, which down-regulated in DG (Figure 3). PPAR- $\gamma$ is widely distributed in the adipocytes to help to enhance insulin sensitivity (Kahn et al., 2010) and have a vital function in reducing fatty acid blood concentration; thus regulate lipid metabolism (Lee et al., 2010). This study showed that GBE and PGE in combination have synergistic effects to improve dyslipidemia and hyper-cholesterolemia by regulating the expression of SREBP-1c and FAS (liver), PPAR- $\alpha$ (muscle) and PPAR- $\gamma$ (adipose tissues) of diabetic rats.

Determination of anti-oxidative activities: A significant increase for MDA $(p<0.0001)$ and a significant decrease for CAT $(\mathrm{p}<0.0001)$ serum concentrations were found in diabetic rats. However, we recorded a significant decrease for MDA both in MG1, MG2 $(p<0.001)$, and MG3 $(p<0.0001)$ and a significant increase for serum CAT $(\mathrm{p}<0.05)$ in all the three treated groups (Table 3). Scientific evidence showed that oxidative stress caused chronic hyper-glycemia in diabetes thus reducing anti-oxidant defense properties and if remain untreated might promote the generation of free radicals and cause various health problems (Jung et al., 2005). Our data showed that GBE and PGE overcome oxidative stress and have strong anti-oxidative properties. The anti-oxidative activities of ginkgo are attributed to flavonoids (Maltas and Yaldiz, 2012) and that of ginseng is ginsenosides (Murphy and Lee, 2002).

Down-regulation of TNF- $\alpha$ by GBE and PGE: Down regulation of MG2 $(p<0.001)$ and MG3 $(p<0.0001)$ in muscles (Figure 1), of MG3 $(\mathrm{p}<0.05)$ in hepatic tissues (Figure 2) and down-regulation $(\mathrm{p}<0.0001)$ in all treated groups in adipose tissues (Figure 3 ) was observed, which previously found to be up-regulated in diabetic rats. Results are consistent with (Naseem et al., 2016) for ginkgo and (Jeon et al., 2013) for ginseng on mRNA expression of TNF- $\alpha$. TNF- $\alpha$; plays a vital role in the inflammatory response. In obesity; the up-regulation of adipocyte TNF- $\alpha$ increases the chance of diabetes by increasing $\beta$-cells apoptosis (Badawi et al., 2010).

Determination of Biochemical Parameters: Serum AST, ALT, and Creatinine concentrations are given in Table 3. A significant decrease for serum AST was found in MG2 $(p<0.05)$, and MG3 $(p<0.0001)$, whereas, we recorded a significant reduction for ALT only for MG3 $(p<0.05)$. Furthermore, a significant decrease was found for MG1 $(p<0.05)$ and MG3 $(p<0.05)$ for serum creatinine levels. The stability of AST, ALT, and creatinine showed protecting the effect of GBE and PGE against toxic properties of alloxan.

Liver enzymes (AST and ALT) are important bio-marker to identify liver diseases; and literature supports the arguments that these said enzymes are linked to the development of diabetic complications (Kunutsor et al., 2013). High creatinine concentration in blood is an indicator of renal dysfunction (Salih, 2012). 
Table 1. Genes and the primer sequences used in this study.

\begin{tabular}{cll}
\hline Genes & \multicolumn{1}{c}{ Forward primer (5'-3') } & \multicolumn{1}{c}{ Reverse primer (5'-3') } \\
\hline GAPDH & "TCCCATTCTTCCACCTTTGATGCT" & "ACCCTGTTGCTGTAGCCATATTCAT" \\
GLUT4 & "GCTTCTGTTGCCCTTCTGTC" & "TGGACGCTCTCTTTCCAACT" \\
IR & "GTGCTGCTCATGTCCTAAGA" & "AATGGTCTGTGCTCTTCGTG" \\
IRS-1 & "GCCAATCTTCATCCAGTTGC" & "CATCGTGAAGAAGGCATAGG" \\
SREBP-1c & "GGCATGAAACCTGAAGTGGT" & "TGGGCTTTCACCTGGTTATC" \\
FAS & "CGCCGTGGTGCTGGAGATTG" & "CTTGCCGAGGTTGGTGAGGAAG" \\
PPAR- $\alpha$ & "GAGACCCTCGGGGATCTTAG" & "CGTCTTGTGTCCTGAGCTTG" \\
PPAR- $\gamma$ & "CTGACCCAATGGTTGCTGATTAC" & "GGACGCAGGCTCTACTTTGATC" \\
TNF- $\alpha$ & "GCAGAGCCTTCCAAGCCTACC" & "GTTACCCAGCCCACCTCCTTTG"
\end{tabular}

Abbreviations: Glyceraldehyde 3 Phosphate Dehydrogenase (GAPDH), Glucose Transporter- 4 (GLUT-4), Insulin Receptor (IR), Insulin Reseptor Substrate-1 (IRS-1), Sterol Regulatory Element Binding Protein-1c (SREBP-1c), Fatty Acid Synthase (FAS), Peroxisome Proliferator-Activated Receptor- $\alpha$ (PPAR- $\alpha$ ), Proliferator-Activated Receptor- $\gamma$ (PPAR- $\gamma$ ), Tumor Necrosis Factor- $\alpha$ $(\mathrm{TNF}-\alpha)$

Table 2. Synergistic effects of Ginkgo biloba leaf extract (GBE) and Panax ginseng root extract (PGE) on body weight and blood glucose concentration at week-1 and week-14 in alloxan induced diabetic rats $(\mathrm{N}=8)$..

\begin{tabular}{|c|c|c|c|c|c|c|c|c|c|c|}
\hline \multirow[t]{2}{*}{ Parameters } & \multicolumn{2}{|c|}{ NDG } & \multicolumn{2}{|c|}{ DG } & \multicolumn{2}{|c|}{ MG 1} & \multicolumn{2}{|c|}{ MG 2} & \multicolumn{2}{|c|}{ MG 3} \\
\hline & Wk-1 & Wk- 14 & Wk-1 & Wk-14 & Wk-1 & Wk-14 & Wk-1 & Wk-14 & Wk-1 & Wk-14 \\
\hline Body & 165.36 & 206.20 & 165.93 & 159.08 & 165.98 & 159.08 & 166.91 & 163.93 & 166.76 & 160.08 \\
\hline weight (g) & \pm 1.51 & $\pm 2.36^{\mathrm{a}}$ & \pm 1.14 & $\pm 2.53^{c}$ & \pm 1.37 & $\pm 2.53^{\mathrm{b}}$ & \pm 1.53 & $\pm 3.14^{\mathrm{b}}$ & \pm 1.16 & $\pm 1.94^{\mathrm{b}}$ \\
\hline Blood & 83.43 & 83.68 & 417.07 & 485.58 & 412.81 & 178.05 & 404.96 & 178.87 & 388.86 & 151.37 \\
\hline $\begin{array}{l}\text { Glucose } \\
\text { (mg/dl) }\end{array}$ & $\pm 1.28^{\mathrm{b}}$ & $\pm 1.35^{\mathrm{c}}$ & $\pm 25.43^{\mathrm{a}}$ & $\pm 25.52^{\mathrm{a}}$ & $\pm 15.28^{\mathrm{a}}$ & $\pm 1.63^{\mathrm{b}}$ & $\pm 7.75^{\mathrm{a}}$ & $\pm 1.22^{\mathrm{b}}$ & $\pm 7.05^{\mathrm{a}}$ & $\pm 3.05^{\mathrm{b}}$ \\
\hline
\end{tabular}

Data represented as Mean \pm SEM. Superscripts ${ }^{\mathrm{a}-\mathrm{e}}$ represent significant variation between groups in rows $(\mathrm{p}<0.05)$.

Table 3. Synergistic effects of GBE and PGE on Bio-chemical and lipid profile fasting in alloxan induced diabetic rats $(\mathbf{N}=8)$.

\begin{tabular}{|c|c|c|c|c|c|}
\hline Parameters & NDG & DG & MG1 & MG2 & MG3 \\
\hline FSG (mg/dl) & $83.68 \pm 1.35^{\mathrm{c}}$ & $485.58 \pm 25.52^{\mathrm{a}}$ & $178.05 \pm 1.63^{\mathrm{b}}$ & $178.87 \pm 1.22^{\mathrm{b}}$ & $162.55 \pm 0.91^{\mathrm{b}}$ \\
\hline $\mathrm{TC}(\mathrm{g} / \mathrm{L})$ & $0.82 \pm 0.01^{\mathrm{c}}$ & $1.34 \pm 0.01^{\mathrm{a}}$ & $1.29 \pm 0.01^{\mathrm{ab}}$ & $1.32 \pm 0.01^{\mathrm{a}}$ & $1.25 \pm 0.02^{\mathrm{b}}$ \\
\hline VLDL-c (g/L) & $0.07 \pm 0.005^{\mathrm{e}}$ & $0.28 \pm 0.005^{\mathrm{a}}$ & $0.26 \pm 0.01^{\mathrm{b}}$ & $0.22 \pm 0.002^{\mathrm{c}}$ & $0.18 \pm 0.003^{\mathrm{d}}$ \\
\hline LDL-c (g/L) & $0.06 \pm 0.001^{\mathrm{d}}$ & $0.55 \pm 0.01^{\mathrm{a}}$ & $0.43 \pm 0.004^{b}$ & $0.43 \pm 0.005^{b}$ & $0.30 \pm 0.005^{\mathrm{c}}$ \\
\hline HDL-c (g/L) & $0.68 \pm 0.01^{\mathrm{b}}$ & $0.49 \pm 0.009^{\mathrm{e}}$ & $0.54 \pm 0.01^{\mathrm{d}}$ & $0.647 \pm 0.007^{\mathrm{c}}$ & $0.76 \pm 0.01^{\mathrm{a}}$ \\
\hline TG (g/L) & $1.25 \pm 0.007^{\mathrm{c}}$ & $2.12 \pm 0.02^{\mathrm{a}}$ & $1.38 \pm 0.03^{b}$ & $1.28 \pm 0.01^{\mathrm{c}}$ & $1.23 \pm 0.01^{\mathrm{c}}$ \\
\hline CAT (KU/L) & $20.83 \pm 0.26^{\mathrm{a}}$ & $18.60 \pm 0.65^{\mathrm{b}}$ & $19.55 \pm 0.13^{\mathrm{b}}$ & $19.50 \pm 0.12^{\mathrm{b}}$ & $19.49 \pm 0.16^{\mathrm{b}}$ \\
\hline MDA (mmol/L) & $6.54 \pm 0.23^{\mathrm{b}}$ & $7.55 \pm 0.28^{\mathrm{a}}$ & $6.35 \pm 0.21^{\mathrm{b}}$ & $6.34 \pm 0.11^{\mathrm{b}}$ & $6.32 \pm 0.11^{\mathrm{b}}$ \\
\hline $\operatorname{AST}(\boldsymbol{\mu} / \mathbf{L})$ & $76.88 \pm 1.43^{c}$ & $210.06 \pm 3.08^{\mathrm{a}}$ & $199.66 \pm 0.36^{\mathrm{ab}}$ & $195.28 \pm 1.15^{\mathrm{ab}}$ & $194.3 \pm 12.23^{b}$ \\
\hline $\operatorname{ALT}(\boldsymbol{\mu} / \mathbf{L})$ & $36.23 \pm 0.72^{\mathrm{c}}$ & $40.9 \pm 1.68^{a}$ & $39.48 \pm 0.64^{\mathrm{ab}}$ & $39.96 \pm 0.47^{\mathrm{ab}}$ & $37.58 \pm 0.61^{\mathrm{bc}}$ \\
\hline Creatinine (mg/dl) & $1.63 \pm 0.12^{\mathrm{b}}$ & $2.01 \pm 0.03^{\mathrm{a}}$ & $1.75 \pm 0.06^{\mathrm{b}}$ & $1.82 \pm 0.04^{\mathrm{ab}}$ & $1.7 \pm 0.03^{\mathrm{b}}$ \\
\hline
\end{tabular}




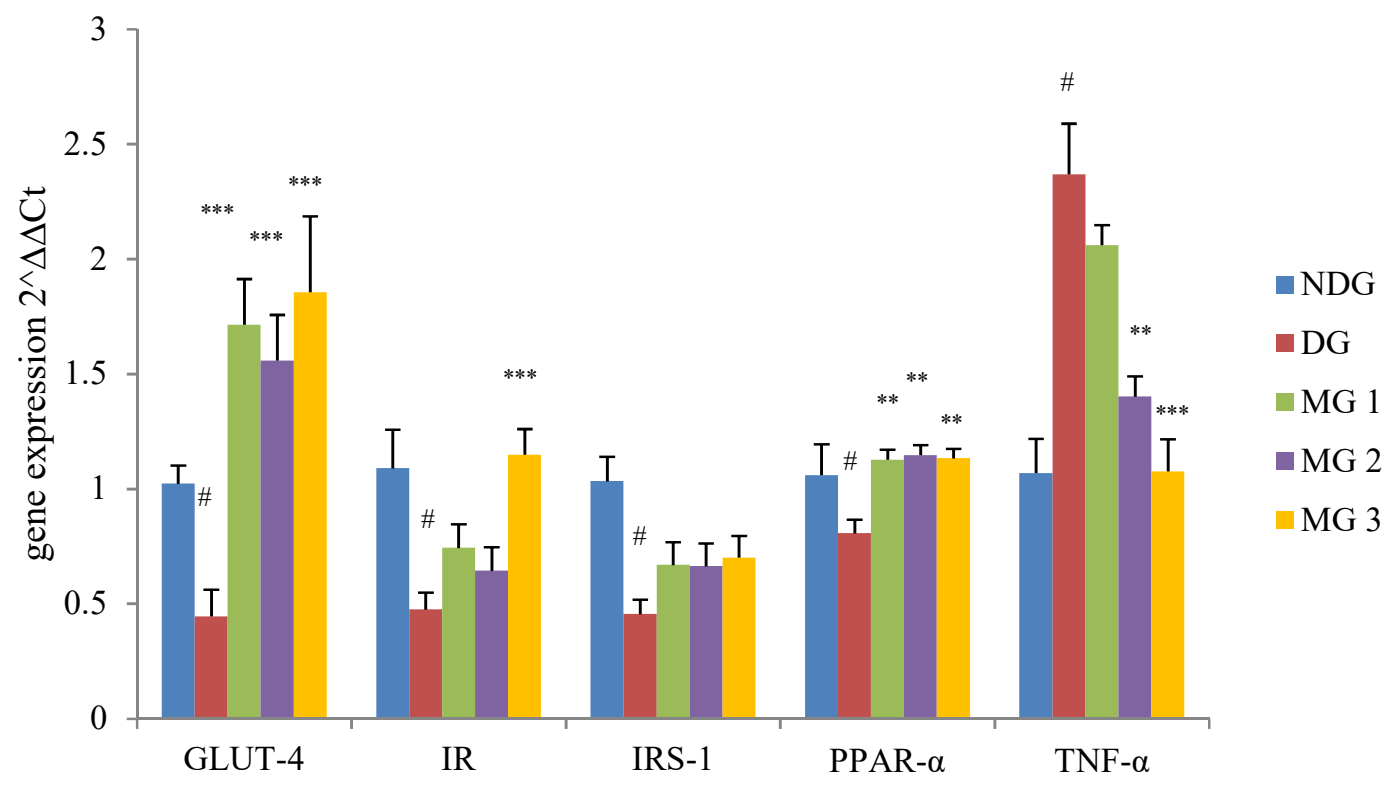

Figure 1. Synergistic effects of GBE and PGE on mRNA expression of GLUT-4, IR, IRS-1, PPAR- $\alpha$ and TNF- $\alpha$ genes in skeletal muscles of non-diabetic (NDG), diabetic (DG), mixed group-1 (MG1), mixed group-2 (MG2) and mixed group-3 (MG3) in alloxan-induced diabetic rats $(\mathrm{N}=8)$. Data represented as Mean \pm SEM. *indicates comparison among DG and NDG, *indicates comparison among treatment groups and DG. * stands for $\mathbf{P}<0.05, * * \mathbf{P}<0.001$ and $* * * \mathbf{P}<0.0001$.

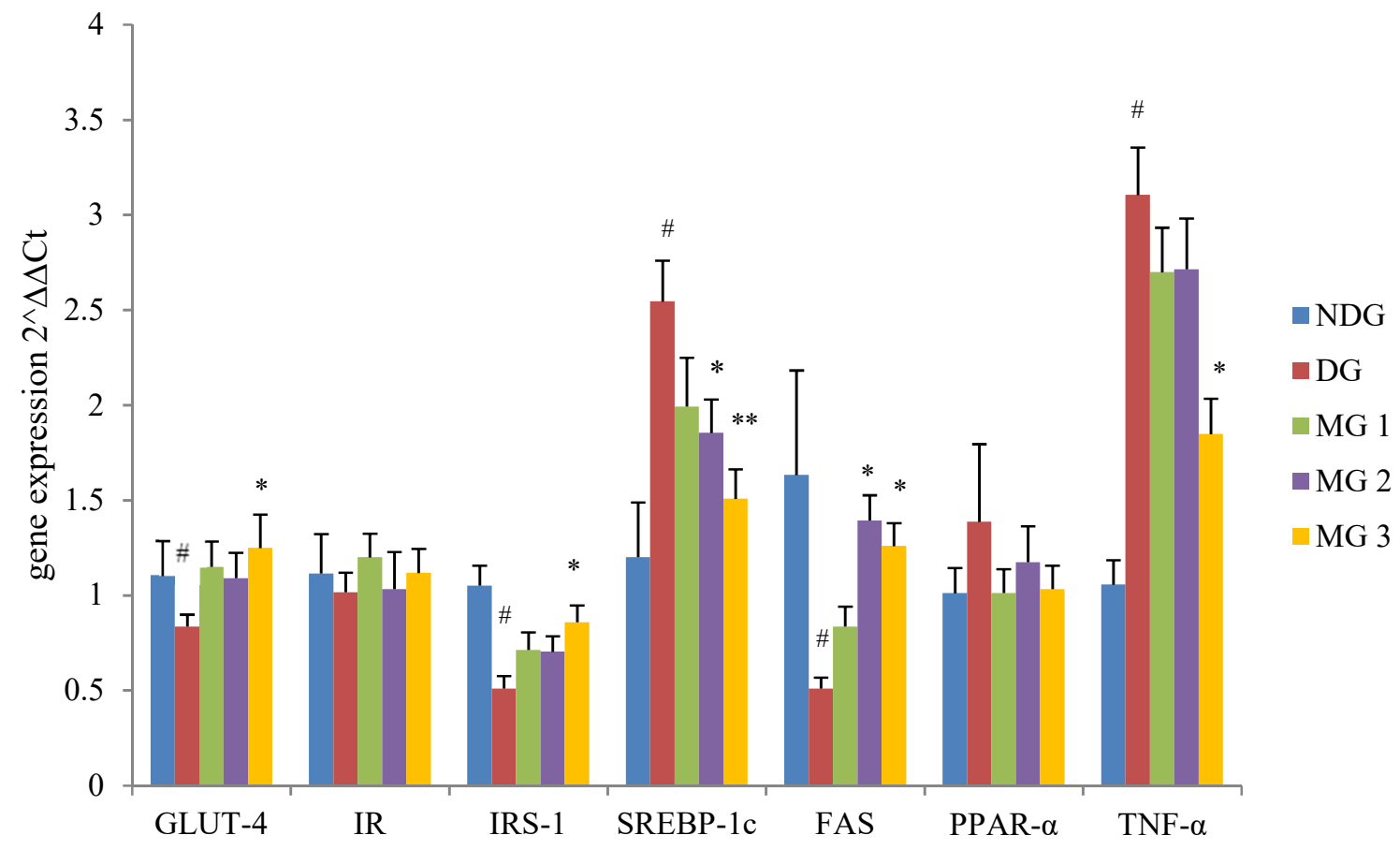

Figure 2. Synergistic effects of GBE and PGE on mRNA expression of GLUT-4, IR, IRS-1, SREBP-1c, FAS, PPAR- $\alpha$ and TNF- $\alpha$ genes in hepatic tissues of non-diabetic (NDG), diabetic (DG), mixed group-1 (MG1), mixed group-2 (MG2) and mixed group-3 (MG3) in alloxan-induced diabetic rats (N=8). Data represented as Mean \pm SEM. \#indicates comparison among DG and NDG, "indicates comparison among treatment groups and DG. * stands for $\mathbf{p}<0.05$ and ** $p<0.001$ 


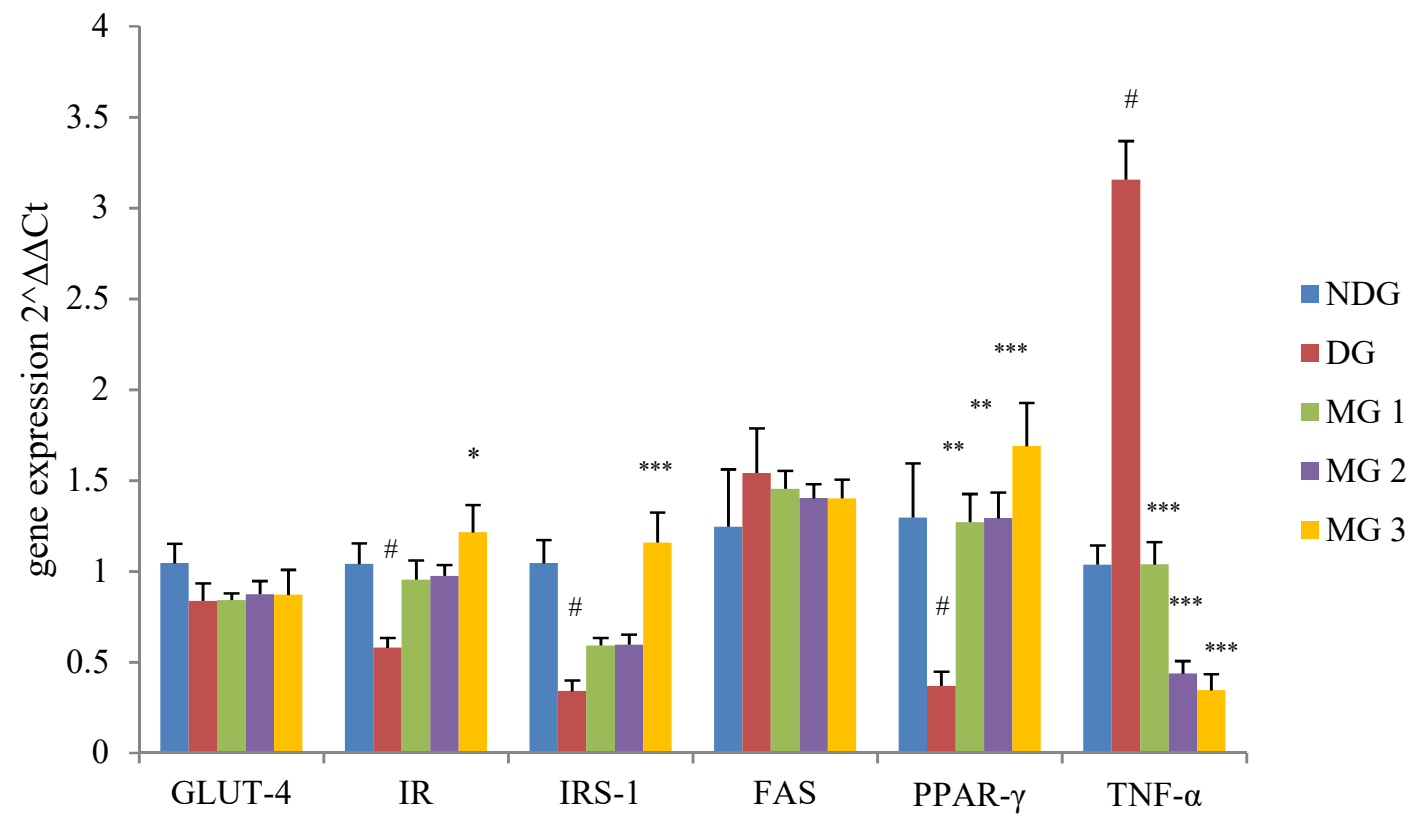

Figure 3. Synergistic effects of GBE and PGE on mRNA expression of GLUT-4, IR, IRS-1, FAS, PPAR- $\gamma$ and TNF- $\alpha$ genes in adipose tissues of non-diabetic (NDG), diabetic (DG), mixed group-1 (MG1), mixed group-2 (MG2) and mixed group-3 (MG3) in alloxan-induced diabetic rats (N=8). Data represented as Mean \pm SEM. \#indicates comparison among DG and NDG, *indicates comparison among treatment groups and DG. *stands for $\mathbf{p}<0.05, * * \mathbf{p}<0.001$ and $* * * \mathbf{p}<0.0001$.

Conclusion: In conclusion, we found, that GBE and PGE in combination showed synergistic effects depending upon doses. The combination of both herbal extracts showed effective anti-hyperglycemic effects by upregulated GLUT-4 and IRS-1 in hepatic tissues; GLUT-4 and IR in skeletal muscle; IR and IRS-1 in adipose tissue, anti- hypercholesterolemia by up-regulating the expression of FAS in hepatic tissues, antihypertriglyceridemic by up-regulation SREPBP-1c in hepatic tissues; PPAR- $\alpha$ in skeletal muscle and PPAR- $\gamma$ in adipose tissue, anti-oxidative by decreasing MDA and increasing CAT serum level and in addition to these effects down-regulated the expression of TNF- $\alpha$ in studied organs in alloxan-induced diabetic rats.

Acknowledgments: This project was funded by the Higher Education Commission (HEC, Pakistan) and West Human Nutrition Research Center (France) for providing all laboratory facilities for the execution of this research.

\section{REFERENCES}

Alam, M.M., D. Merza, and I. Naseem (2014). Protective effect of quercetin on hyperglycemia, oxidative stress and DNA damage in alloxan induced type 2 diabetic mice. Life Sci. 109(1): 8-14.

Amin, K.A., E.M. Awad, and M.A. Nagy (2011). Effects of Panax quinquefolium on Streptozotocininduced diabetic rats: role of $\mathrm{C}$-peptide, nitric oxide and oxidative stress. Int. J. Clin. Exp. Med. 4(2): 136-147.

Badawi, A., A. Klip, P. Haddad, D.E.C, Cole, B.G. Bailo, A. El-Sohemy, and M. Karmali (2010). Type 2 diabetes mellitus and inflammation prospects for biomarkers of risk and nutritional intervention. Diabetes Metab. Syndr. Obes. 3:173-186.

Baynes, C., A. D. Henderson, V. Anyaoku, W. Richmond, C.L. Hughes, D.S. Johston, and R. S. Elkeles (1991). The role of insulin sensitivity and hepatic lipase in the dyslipidaemia of type 2 diabetes. Diabet. Med. 8(6): 560-566.

Calle, C., B. Maestro, and M.G. Arencibia (2008). Genomic actions of 1, 25 dihydroxyvitamin D3 on insulin receptor gene expression, insulin receptor number and insulin action in the kidney, liver and adipose tissues of streptozotocin-induced diabetic rats. Mol. Biol. 9:65-77.

Cheng, D., B. Liang, and Y. Li (2012). Antihyperglycemic effect of Ginkgo biloba extract in Streptozotocin-induced diabetes in rats. Biomed. Res. Inter. 2013:1-7.

Cheon, J. M., D. I. Kim, and K. S. Kim. (2015). Insulin sensitivity improvement of fermented Korean Red Ginseng (Panax ginseng) mediated by insulin resistance hallmarks in old-aged ob/ob mice. J. Ginseng Res. 39, 331-337. 
Cho, W.C.S., W. Chung, S.K.W. Lee, A.W.N. Leung, C.H.K. Chen, and K.K.M. Yue (2006). Gisenoside Re of Panax ginseng possesses significant antioxidant and antihyperlipidemic efficacies in streptozotocin induced diabetic rats. Eur. J. Pharmocol. 550(1): 173-279.

Ebuehi, O.A.T., A.E. Ajuluchukwu, O.T. Afolabi, and A.I. Akinwande (2010). Oxidative stress in alloxan-induced diabetes in female and male rats. Adv Int Med. Dent. Sci. 3(3): 71-75.

El-Karim, D. R. G., S. S. Oda, H. G. Tohamy, and M. A. Hashem (2017). The effect of metformin and ginseng on alloxan-induced diabetic rats: hematological, biochemical and histopathological studies. Alex. J. Vet. Sci. 55(1): 60-66.

Horton, J.D., J.L. Goldstein, and M.S. Brown. (2002). SREBPs: activators of the complete program of cholesterol and fatty acid synthesis in the liver. J. Clin. investing. 109(9): 11251131.

Huang, S., and M.P. Czech (2007). The GLUT-4 glucose transporter. Cell Metab. 5(4):237-252.

Jeon, W.J., J. S. Oh, M.S. Park, and G.E. Ji (2013). Antihyperglycemic effect of fermented ginseng in type 2 diabetes mellitus mouse model. Phytother. Res. 27(2): 166-172.

Jung, C., H.M. Seog, I.W. Choi, H.D.Choi, and H.Y. Cho (2005). Effects of wild ginseng (Panax ginseng C.A. Meyer) leaves on lipid peroxidation levels and antioxidant enzyme activities in streptozotocin diabetic rats. J. Ethnopharmacol. 98(3): 245-250.

Kahn, S.E., S.M. Haffner, G. Viberti, W.H. Herman, J.M. Lachin, B.G. Kravitz, D. Yu, G. Paul, R.R. Holman, and B. Zinman (2010). Rosiglitazone decreases C-reactive protein to a greater extent relative to glyburide and metformin over 4 years despite greater weight gain: observations from a Diabetes Outcome Progression Trial (ADOPT). Diabetes Care. 33(1): 177-183.

Kamel, E. O., and A. S. A. H. Abd-Elrhman. (2018). The effect of diabetes mellitus on the rat ventral prostate and the possible protective role of Ginkgo biloba extracts. Al-Azhar Assiut. Med. J, 16(3): 300 .

Kim, Y.J., J.N. Jeon, M.G. Jang, J.Y. Oh, W.S. Kwon, S.K. Jung, and D.C. Yang (2014). Ginsinoside profiles and related gene expression during foliation in Panax ginseng Meyer. J. Ginseng Res. 38(1): 66-72.

Krishnamurthy, G., K. Lakshman, N. Pruthvi, and P.U. Chandrika (2011). Antihyperglycemic and hypolipidemic activity of methanolic extract of Amaranthus viridis leaves in experimental diabetes. Ind. J. Pharmacol. 43(4): 450-454.
Kumar, R. (2012). Correlation of Selenium and other antioxidants in diabetic patients with and without complications. Free Rad Antiox. 2 (1): 6-8.

Kunutsor, S.K., T.A. Apekey, and J, Walley (2013). Liver aminotransferase and risk of the incident type 2 diabetes: A systematic review and Metaanalysis. Am. Epidemiol. 178(2): 159-171.

Lee, S.H., H.J. Lee, Y.H. Lee, B.W. Lee, B.S. Cha, E.S. Kang, C.W. Ahn, J.S. Park, H.J. Kim, E.Y. Lee, and H.C. Lee (2012). Korean red ginseng (Panax ginseng) improves insulin sensitivity in high fat fed Sprague-Dawley rats. Phytother. Res. 26(1): 142-147.

Lee, Y.S., B.Y. Cha, K. Saito, H. Yamakwa, S.S. Choi, K. Yamaguchi, T. Yonezawa, T. Teruya, K. Nagai, and J.T. Woo (2010). Nobiletin improves hyperglycemia and insulin resistance in obese diabetic ob/ob mice. Biochem. Pharm. 79(11): 1674-1683.

Leto, D., and A.R. Saltiel (2012). Regulation of glucose transport by insulin: traffic control of GLUT-4. Natl. Rev. Mol. Cell. Biol. 13(6): 383-396.

Lin, E., Y. Wang, S. Mehendale, S. Sun, C.Z. Wang, J.T. Xie, H.H. Aung, and C.S. Yuan (2008). Antioxidant protection by American ginseng in pancreatic $\beta$-cells. Am. J. Chin. Med. 36(5): 981-988.

Maltas, E, and S. Yildiz (2012). Evaluation of phytochemicals and antioxidant activity of Ginkgo biloba from Turkey. Pharmacologia. 3(4): 113-120.

Menendez, J.A., A.V.Martin, F.J. Ortega, and J.M.F. Real (2009). Fatty acid synthase: Associated with insulin resistance, type 2 diabetes and cancer. Clin. Chem. 55(3): 425-438.

Murphy, L.L., and T.J. Lee (2002). Ginseng, sex behavior and nitric oxide. Ann. Acad. Sci. 962: 372-377.

Naseem, M., M.Q. Zaman, H. Nazih, K. Ouguerram I. Rabbani, H. Zaneb T. Yaqoob, H.U. Rehman, J. Michel, S.K. Tahir, M. S. Yousaf, K. A. Majeed, $\mathrm{K}$ and M.S. Hussain (2016). The effects of ginkgo biloba leaf extract on metabolic disturbances associated to alloxan-induced diabetic rats. J. Anim. And Plant Sci. 26(3): 627 635.

Ramadan, G., N.M. El-Beih, and A.E.A. El-Ghaffar (2009). Modulatory effects of the black V. green tea aqueous extract on hyperglycemia, hyperlipidaemia and liver dysfunction in diabetic and obese rat models. Br. J. Nutr. 102(11): 1611-1619.

Salih, N.A. (2012). Effect of ginseng (Panaxginseng) on experimentally induced diabetes mellitus in male rabbits. Al-Anbar. J. Vet. Sci. 5(1): 188194. 
Smith, J. V., and Y, Luo. (2003). Elevation of oxidative free radicals in Alzheimer's disease models can be attenuated by Ginkgo biloba extract EGb 761. J. Alzheimer's Dis. 5(4): 287-300.

Swanston-Flatt, S.K., C. Day, C.J. Bailey, and P.R. Flatt (1990). Traditional plant treatments for diabetes. Study in normal and streptozotocin diabetic mice. Diabetologia. 33(8): 462-464.

Taniguchi, C,M., K. Veki and C.R. Kahn (2005). Complementary roles of IRS-1 and IRS-2 I the hepatic regulation of metabolism. J. Clin. Invest. 115: 781-727.

Unger, M. (2013). Pharmocokinetic drug interactions involving Ginkgo biloba. Drug Metab. Rev. 45(3): 353-385.

Vuksan, V., Z. Z. Xu, E, Jovanovski, A. L. Jenkins, U. Beljan-Zdravkovic, J. L. Sievenpiper, P. M. Stavro, A. Zurbau, L. Duvnjak, and M. Z. Li. (2019). Efficacy and safety of American ginseng (Panax quinquefolius L.) extract on glycemic control and cardiovascular risk factors in individuals with type 2 diabetes: a double-blind, randomized, cross-over clinical trial. Eur. J. Nutr. 58(3): 1237-1245.

Xie, J.T., Y.P. Zhou, L. Dey, A.S. Attele, J.A. Wu, M. Gu, K.S. Polonsky, and C.S. Yuan (2002). Ginseng berry reduces blood glucose and body weight in $\mathrm{db} / \mathrm{db}$ mice. Phytomedicine. 9(3): 254258.

Yoon, M., M. Lee, S. Jeong, J.J. Kim, C.J. Nicol, K.W. Nam, M. Kim, B.G. Cho, and G.T. Oh (2003). Peroxisome proliferator-activated receptor $\alpha$ is involved in the regulation of lipid metabolism by ginseng. Br. J. Pharmacol. 138(7): 12951302.

Yuan, H.D., S.J. Kim, and S.H. Chung (2011). Beneficial effects of IH-901 on glucose and lipid metabolisms via activating adenosine monophosphate activated protein kinase and phosphatidylinositol-3 kinase pathways. Metab. Clin. Exp. 60(1): 43-51.

Zhou, L., Q. Meng, T. Qian, and Z. Yang (2011). Ginkgo biloba extract enhances glucose tolerance in hyperinsulinisim induced hepatic cells. J. Nat. Med. 65(1): 50-56. 